\title{
Minimizing CoAting Process Sourced HYDROGEN EMBRITTLEMENT OF FASTENERS
}

\author{
Ugur Buyuk, Anil Akdogan, Ali Serdar Vanli \& Numan Durakbasa
}
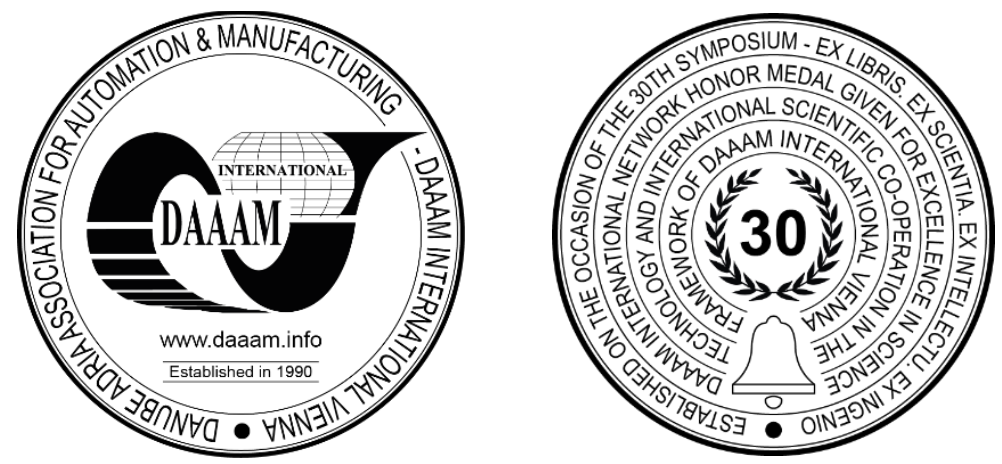

This Publication has to be referred as: Buyuk, U[gur]; Akdogan, A[nil]; Vanli, A[li] S[erdar] \& Durakbasa, N[uman] (2021). Minimizing Coating Process Sourced Hydrogen Embrittlement of Fasteners, Proceedings of the 32nd DAAAM International Symposium, pp.0508-0513, B. Katalinic (Ed.), Published by DAAAM International, ISBN 978-3-90273433-4, ISSN 1726-9679, Vienna, Austria

DOI: $10.2507 / 32$ nd.daaam.proceedings.073

\begin{abstract}
Fasteners are devices that mechanically fix two or more objects to each other used in many joints. The phenomenon of hydrogen embrittlement can be very common in fastener materials, causing problems in the assembly of fasteners and reducing their mechanical properties. Hydrogen embrittlement means the embrittlement of a metal by diffusible hydrogen due to the absorption of its atoms or molecules by the material. Appropriate plating or coating processes should be used in the surface coating of fasteners to minimize hydrogen embrittlement. In this paper, we examined spring type straight pins as fasteners to be coated to avoid unexpected failures due to hydrogen diffusion such as low mechanical properties. Choosing the right coating type and conditions improved assembly productivity and the performance of spring type straight pins.
\end{abstract}

Keywords: Fasteners; Coating; Hydrogen embrittlement; Mechanical properties

\section{Introduction}

Fasteners are hardware tools which mechanically joins two or more objects together. Fasteners usually being used to create temporary joints; that is, joints that can be removed or disassemble without damaging the joining parts. It is overcritical to choose the right fasteners for production. First, it should be suitable for parts and applications. Additionally, there are two important decisions to take at design stage.

- Selecting the most appropriate material,

- Applying the right coating operation.

Designers have a simple and efficient way when selecting materials in the design phase to prevent damage and defect in structural materials throughout the process lifetime and occasionally further on to improve to application life. They must know if a material is susceptible to cracking. In addition, quick decisions or process measures are often obligatory throughout service time, to prevent or delay this type of damage which is hard situation [1].

Sometimes it is not enough to choose a suitable material for mechanical durability and a suitable coating process against corrosion to achieve good performance. It is essential to establish a relation between material and coating process. Hydrogen embrittlement can occur if the material and coating process do not suitable to each other. 
Unfortunately, it effects mechanical properties of the material in a negative way. Hydrogen embrittlement means the embrittlement of a metal by diffusible hydrogen due to the absorption of its atoms or molecules by the material. In many studies, hydrogen can affect crack distribution, such as stress corrosion cracking and corrosion fatigue. Three situations are imperative to establish crack initiation that can lead to failure. The first is the existence of hydrogen, the second is the tensile stresses and the last is the sensitivity of the material [2]. The phenomenon of hydrogen embrittlement can be very common in fasteners and cause problems during assembly. The selected proper coating type improved assembling productivity and performance of spring type straight pins.

Spring Type Straight Pin is common fastener which could be used on many applications. There is an international standard numbered ISO 8752 about this hard situ. Spring Type Straight Pin has a special flexibility according to the standard. It can easily fix to hole which has suitable dimension defined by ISO 8752 . Figure 1 determines the geometry of spring type straight pin, and the Figure 2 defines proper dimension of the hole. Appropriate plating or coating processes are needed to avoid hydrogen embrittlement if the pins are surface coated. The pins shouldn't be electroplated or phosphate-coated due to the risk of hydrogen embrittlement. If electroplating or phosphate plating is required for corrosion prevention, it is obligatory that the pins be thermally treated immediately after plating to minimize the risk of hydrogen embrittlement. Nevertheless, preventing hydrogen embrittlement is not absolutely guaranteed in such processes [3].

This paper details the negative effects of HE in the materials in their usage lifetime and the importance of selecting proper coating type in improvement of assembling productivity and performance of spring type straight pins. It also consists of an example of how hydrogen embrittlement phenomena can be reduced by the right plating process for fasteners.

\section{Hydrogen Embrittlement in Materials}

Hydrogen is the most abundant element in the universe, is safe and clean fuel gas of high calorific value, available in infinite quantities or amounts by the electrolysis of water [4]. High strength materials are too sensitive against hydrogen embrittlement when they stand on hydrogen environment [5]. There are three main reasons which are effect directly hydrogen embrittlement. The first is the stress, the second is the material, and the third is the environmental conditions (Figure 1). When the researchers search for material, they need to consider many variables such as the microstructure, the strength of material, its residual stress, and the applied strain rate. Moreover, the surface conditions and the heat treatments are the other variables. Metallic plating and specific precipitates, applied strain ratio and surface condition of a material, processing pressure, temperature and exposure time, concentration hydrogen and amount of hydrogen trap, solution that make a reaction with metals could be considered as the rest variables.

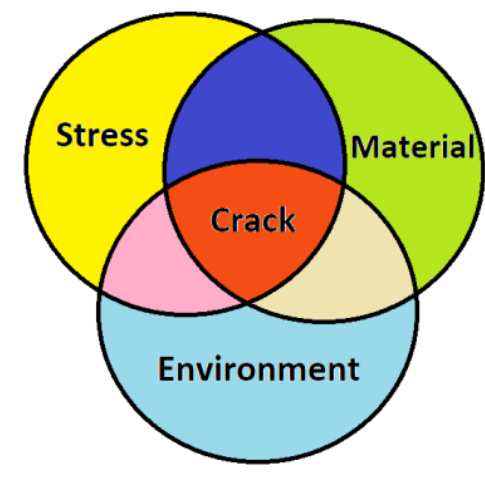

Fig. 1. Three Main Reasons of Hydrogen Embrittlement [6]

Effect of hydrogen embrittling process is the degradation of mechanical properties or delay failure which can occur due to internal residual stresses or applied external stresses. The most mechanical degradation is that normally ductile material become brittle [7]. There are three situations which increase the potential of hydrogen embrittlement. These are high tensile strength, hardness or surface hardening which have could absorbed hydrogen under tensile stress. If the hardness is higher than $320 \mathrm{HV}$, the operation examination shall be using a test to aware of hydrogen embrittlement [8]. If there is any embrittlement, revision of the production operation will be obligatory such as the inclusion of a treatment process. Of course, hydrogen embrittlement prevention cannot be $\% 100$ guaranteed. If a reduced possibility of occurrence hydrogen embrittlement is desired alternative methods should be evaluated. Steels whose strength are more than 1000 $\mathrm{MPa}$ is susceptible for HE [9]

To avoid from HE on high strength materials, the main reason of it and its mechanism must be defined clearly. Choosing of a proper material is crucial. The material should be optimum strength and high resistant alloy for HE. Design of part must be properly detailed. Sharp corners and critical point must be avoided and if any there are any residual stresses, they should be eliminated before the operation. The thermal treatment processes should be applied to detach hydrogen which is consumed and can cause any failure occurs. 
Heat treatment operation conditions must be arranged according to the process performed on the material [10]. Additional cleaning operation should be applied to eliminate some flake and oxide parts from material with the use of acidic solvent. This solution helps to avoid hydrogen diffusion like mechanical means such as sand and grit blasting. Avoid of HE could be done by proper metallic alloys to the base material and applying protective plating, as well [5]. Some plating methods are vacuum deposited, and some are mechanical. Proper inhibitors also help to prevent from HE. Titanium may reduce the HE sensitivity of a hot stamped boron steel by forming titanium carbide in the material if titanium is suitable in a large batch [11].

When atomic hydrogen occurs in steels and other metals like aluminium alloys it can cause loss of ductility or load carrying capability resulting cracking or catastrophic brittle failures at applied stresses well below the yield strength or even the normal design strength for alloys. This situation often occurs in alloys that do not show important loss in ductility when measured by normal tensile tests. This is usually referred to as hydrogen induced postponed brittle failure, hydrogen stress cracking or hydrogen embrittlement. These can be occurred throughout heat treatment, gas carburizing, cleaning, pickling, phosphating, electroplating, autocatalytic operations and in the service environment due to cathodic plating reactions or corrosion due to the break-down of inappropriate lubricants as well as throughout welding or brazing process. There are some parts such as by machined ground cold-formed and hardening heat treatment processed ones more sensitive to hydrogen embrittlement damage [8].

The steps of the production process and its parameters must be checked to eliminate the possibility of embrittlement. Possibility of HE can be reduced during the production process with many ways as mentioned earlier; plating or coating process, baking or heat treatment process, cleaning processes and stress relief. This article examines the possible reasons and solutions of HE in literature and gives an example how hydrogen embrittlement can be reduced by right parameters of plating process for fasteners. Heat-treated or cold worked steels hardened more than $365 \mathrm{HV}$ high cathodic efficiency electroplating solutions are advisable for fasteners. [8]

\section{Case Study}

In this work the designed pin is considered for HE occurrence. When we design our spring-loaded latches, we decide to use slotted spring pin as an examined sample. The technical drawing and photo of the slotted spring pin can be found in Figure 2. ISO 8752 international standard describes the materials for split type straight pins. Suitable slotted spring pin has been chosen by simulation according to the related standard (Table 1). The chosen pin has 5 mm diameter. The tolerances of it given in Table 2.

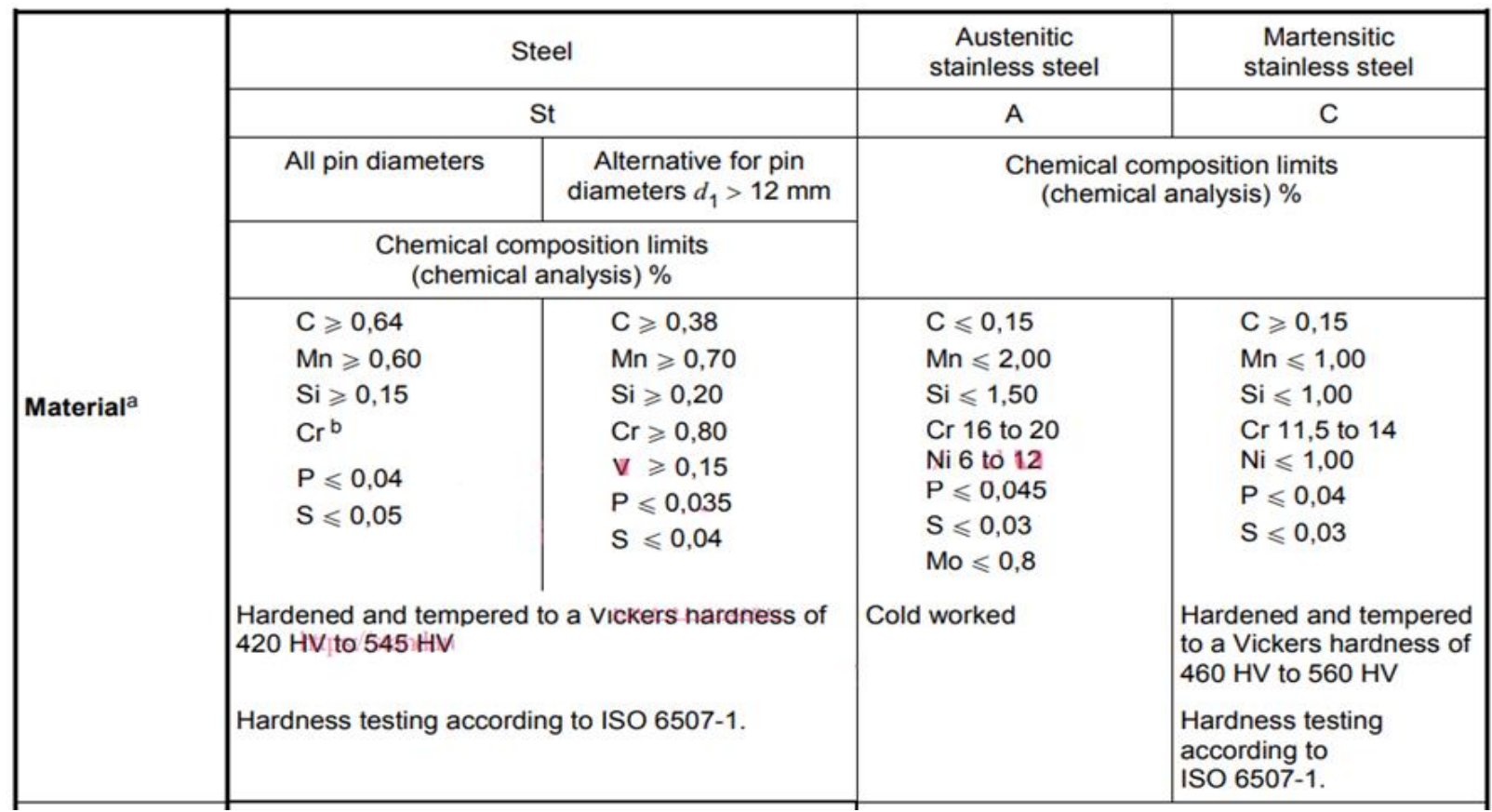

Table 1. Requirements and reference International Standards [3]

\begin{tabular}{|c|c|c|c|c|c|c|c|c|c|c|c|c|c|c|}
\hline & Dimension of Hole & nom. & 1 & 1,5 & 2 & 2,5 & 3 & 3,5 & 4 & 4,5 & 5 & 6 & 8 & 10 \\
\hline \multirow{2}{*}{$d_{1}$} & \multirow{2}{*}{$\begin{array}{l}\text { before } \\
\text { mounting }\end{array}$} & $\max$ & 1,3 & 1,8 & 2,4 & 2,9 & 3,5 & 4,0 & 4,6 & 5,1 & 5,6 & 6,7 & 8,8 & 10,8 \\
\hline & & min. & 1,2 & 1,7 & 2,3 & 2,8 & 3,3 & 3,8 & 4,4 & 4,9 & 5,4 & 6,4 & 8,5 & 10,5 \\
\hline
\end{tabular}

Table 1. Dimension with tolerances [3] 

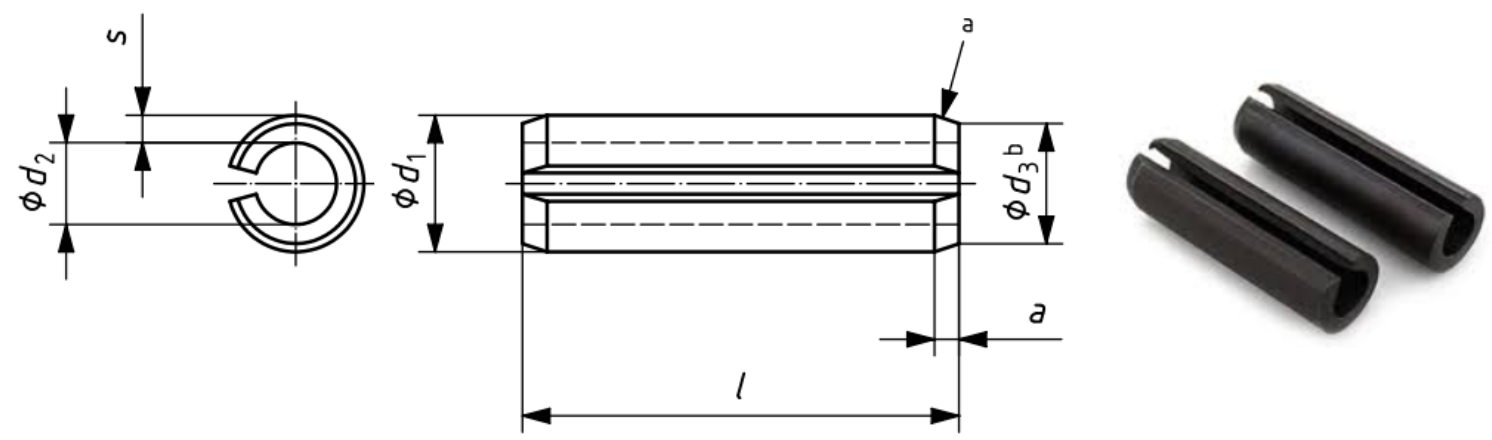

Fig. 2. Slotted Spring Type Straight Pin

The pin is being used on the spring-loaded latches. The slotted pin has been placed on the product via hand press machine as shown in Figure 3. The detailed assembled product shown in Figure 4. While the lock was working open to close position the pin exposes to instant high load. Each position changes of the lock create stress on pin. This cycling effects the lifetime of the examined product.

On the assembling operations the zinc coated pins' sudden cracks could be observed even pins don't have any sign during the process. And it can cause failure cracks during open-close position cycles. It directly effects the product quality and cause customer dissatisfaction. When we assemble the pin from the lock product under the high tensile stress the zinc plated pin getting cracked easily with some reason. When we try the uncoated pin (From same batch) on the product there is no any cracking issue. The Zinc Coating effects pin durability directly. We research the plating to find root cause and we found zinc coating process conditions of us can cause HE phenomena.

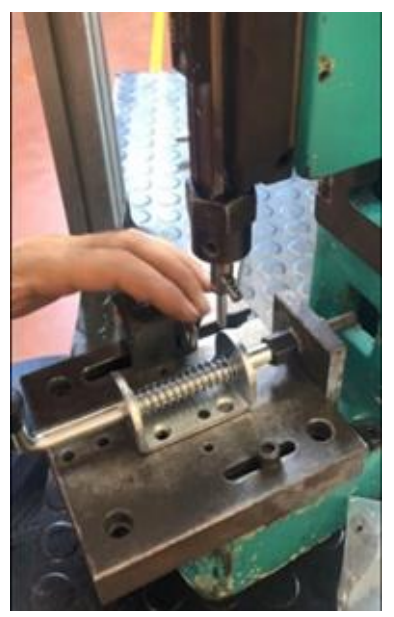

Fig. 3. Assembly operation of the pin

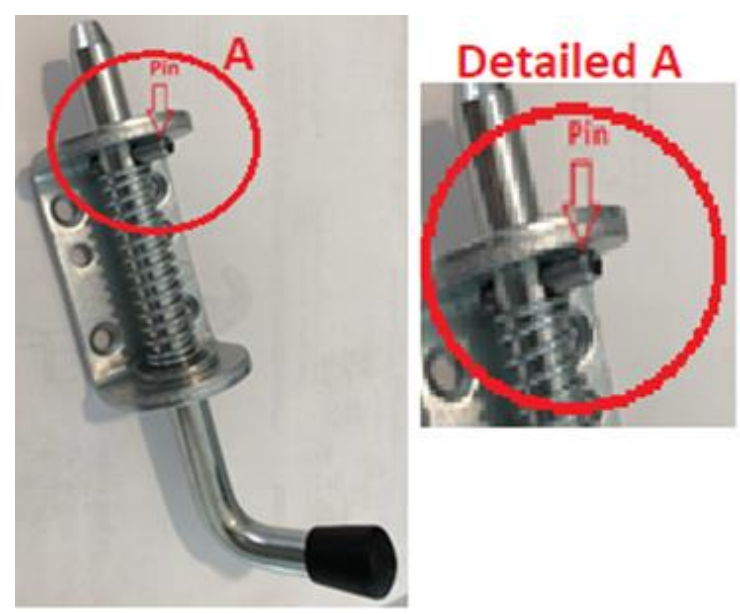

Fig. 4. Assembled Product 
The process of $\mathrm{Zn}$ plating consists of many steps such as cleaning, electroplating, cascade rinsing, bright dip, water rinse, drying, heat treatment, bright dip, water rinse, passivation and finally cascade rinsing. Electroplating is defined as the process or procedure in which a coating is placed by electrolysis, that is, a metal is deposited on an electrode dipped in an electrolyte by passing a suitable electric current through the electrolyte [12]. Zinc plating is not suitable this type of fasteners due to the application process HE. Sometimes, a coating such as graphene coating, CrN, TiAlN coatings etc. are preferred over the other coatings to reduce the effects of the hydrogen embrittlement on high strength material.[12]

We begin by considering other types of coatings. Using a coating process that does not introduce hydrogen into the material (particularly those that do not utilize acids for cleaning) will help avoid this problem A number of dip-spin coatings are considered hydrogen embrittlement "free" because they use mechanical processes (abrasive blasting) for descaling. We found another type of coating that reduce the HE possibility on the item. The pins have been applied two different coating to test and compare. One of them is our classical zinc coating which we have been using already, the other one is a commercial one called Geomet (NOF Metal Coating Asia Pacific CO., LTD. Presented GEOMET® License). This is brand name which invented the special coating by NOF Metal Coating Asia Pacific CO., LTD. Also, similar coatings know by the industry with the name "Zinc Flake Coating".

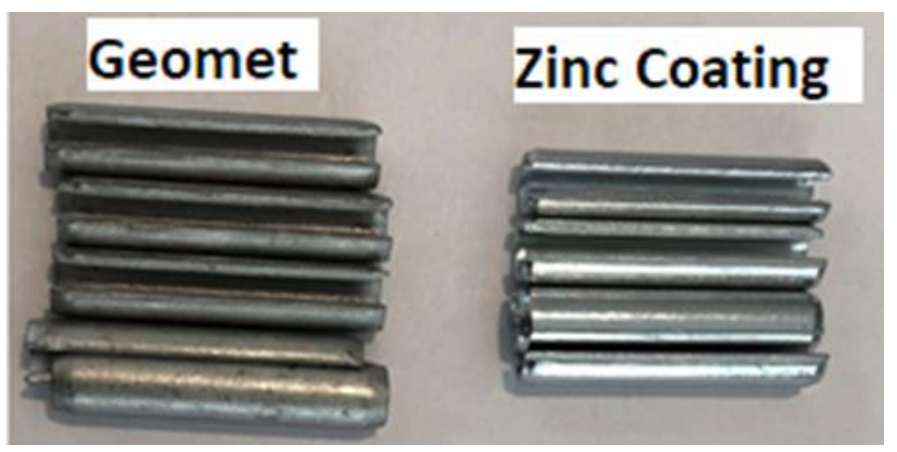

Fig. 5. Visual Check for Zinc \& Geomet Coatings

\begin{tabular}{|c|c|c|c|c|c|}
\hline Thickness & 1.Point $(\boldsymbol{\mu})$ & 2.Point $(\boldsymbol{\mu})$ & 3.Point $(\boldsymbol{\mu})$ & 4.Point $(\boldsymbol{\mu})$ & Average $(\boldsymbol{\mu})$ \\
\hline Zinc Coating & 9,42 & 9,07 & 8,80 & 8,24 & 8,88 \\
\hline Geomet Coating & 11,7 & 11,5 & 9,99 & 14,4 & 11,90 \\
\hline
\end{tabular}

Table 2. Thickness Measurement According Coating Types

After both coating operations the pins was observed at first. The zinc coating looks brighter than the brand mark as seen in Figure 6. Average zinc coating thickness is $8,88 \mu \mathrm{m}$ measured. Average geomet coating thickness is $11,90 \mu \mathrm{m}$ measured The measurement results are given in the Figure 6. When we applied the Geomet on the pin and produce our pilot products, there was not any issue on process of assembly. R\&D started the all the lifespan test on the new plated pin. The performance seems to be satisfied. Cracking failure has been not seen on our products. In the case study show us, when different plating process applied on the same material, it increases HE according with type of coating. It is possible to avoid from $\mathrm{HE}$ with to choose right plating type on it. Geomet coating solved issue of case study, it never come up again.

\section{Conclusions}

Study issue is, it occurs HE problem on fasteners because of appliying wrong type coating. Hardness is a major contributor to hydrogen embrittlement. Harder, stronger materials are more susceptible to failure than weaker, softer ones. In general, if the hardness of the fastener is less than $35 \mathrm{HRC}$, there will probably be little difficulty with hydrogen embrittlement. However, if the fastener has hardness above $40 \mathrm{HRC}$, problems are more likely to occurIt has to been change the coating procees with different type. The other option is to use a fastener which is of a lower hardness. Unfortunately this is not possible to change our hardness because of our application. After research and sample test, we found there was another type of coating. It is named as "Zink Flake". It has coating process that does not introduce hydrogen into the material will help avoid this problem. We continued to our test with Geomet which is a kind of Zinc flake coating.

After our study for coating, we solved the cracking issue with a commercial coating for our specific application. This solution helped to reduce the minimum to occur HE and cracking issues. Also, when we compare the Geomet vs. Zinc coating, we discovered that, the Geomet has many advantages besides to avoid HE. It has better corrosion protection (around the 10 times more) and excellence resistance against some chemical abrasives than usual zinc coating. It needs to be considered HE on the design stage by engineers when they choose their material. There are many ways to prevent from HE. It is crucial to choose right material with suitable coating and correct process. 
This study shows us, how significant is to prevent HE with change to coating type. Product performance effected by all process with right material. These should be made assessment all together, otherwise any disharmony will come up with problem. There are no possibilities to eliminate HE occur totally but it is possible to reduce to potential of HE occurrences via choosing the right material with the right processes. This should be considered especially for high tensile or strength application parts like fasteners. For future, we need to be apply right coating for fasteners on our design phases which their hardeness more than 35. It should be "Zinc Flake" type of coating. First choice will be Geomet for similar example after our case study.

\section{References}

[1] G.Gabetta, P.Cioffi, R.Burschi (2018). Engineering Thoughts On Hydrogen Embrittlement.

[2] Brahimi S. (2014). Fundamentals of Hydrogen Embrittlement in Steel Fasteners Ibeca Tech. Corp.

[3] EN ISO 8752:(2009). Spring-Type Straight Pins Slotted, Heavy Duty.

[4] Cwiek J. (2009). Hydrogen degradation of high-strength steels. Journal of achievements in materials and manufacturing. 2009;37(2):193-212

[5] Sandeep Kumar Dwivedi, Manish Vishwakarma (2018). Hydrogen Embrittlement in Different Materials: A review.

[6] Herring DH (2010). Hydrogen embrittlement wire Form TechnolInt.

[7] Davis JR. (1998). Metals handbook. ASM international.

[8] EN ISO 4042:(2019). Fasteners - Electroplated Coatings.

[9] Venezuela J, Liu Q, Zhang M, Zhou Q, Atrens A. (2016). A review of hydrogen embrittlement of martensitic advanced highstrength steels. Corrosion Rev 2016 Jun 1;34(3):153e86

[10] Grobin Jr AW (1988). Other ASTM committees and ISO committees involved in hydrogen embrittlement test methods. Hydrogen Embrittlement: Prev Contr.

[11] Kim HJ, Jeon SH, Yang WS, Yoo BG, Chung YD, Ha HY, et al. (2018). Effects of titanium content on hydrogen embrittlement susceptibility of hot-stamped boron steels. J Alloy Comp

[12] Amit Kharea, Sandeep Kumar Dwivedib, Dr.Manish Vishwakarmac, Dr.Siraj Ahmedd (2018). Experimental Investigation of Hydrogen Embrittlement during Coating Process and Effect on Mechanical Properties of High Strength Steel used for Fasteners 